\title{
The effect of preoperative nutritional status on postoperative outcomes in children undergoing surgery for congenital heart defects in San Francisco (UCSF) and Guatemala City (UNICAR)
}

\author{
Monique Radman, MD, ${ }^{\text {a,b }}$ Ricardo Mack, MD, ${ }^{\mathrm{d}}$ Joaquin Barnoya, MD, MPH, ${ }^{\text {de, }, \mathrm{f}}$ Aldo Castañeda, MD, PhD, ${ }^{\mathrm{d}}$ \\ Monica Rosales, MD, ${ }^{\mathrm{d}}$ Anthony Azakie, MD ${ }^{\mathrm{c}}$ Nilesh Mehta, MD, ${ }^{\mathrm{g}}$ Roberta Keller, MD, ${ }^{\mathrm{a}}$ \\ Sanjeev Datar, MD, PhD, ${ }^{\mathrm{a}}$ Peter Oishi, MD, ${ }^{\mathrm{a}, \mathrm{b}}$ and Jeffrey Fineman, $\mathrm{MD}^{\mathrm{a}, \mathrm{b}}$
}

Objective: The objective of this study was to determine the association between preoperative nutritional status and postoperative outcomes in children undergoing surgery for congenital heart defects (CHD).

\begin{abstract}
Methods: Seventy-one patients with CHD were enrolled in a prospective, 2-center cohort study. We adjusted for baseline risk differences using a standardized risk adjustment score for surgery for CHD. We assigned a World Health Organization z score for each subject's preoperative triceps skin-fold measurement, an assessment of total body fat mass. We obtained preoperative plasma concentrations of markers of nutritional status (prealbumin, albumin) and myocardial stress (B-type natriuretic peptide [BNP]). Associations between indices of preoperative nutritional status and clinical outcomes were sought.
\end{abstract}

\begin{abstract}
Results: Subjects had a median (interquartile range [IQR]) age of 10.2 (33) months. In the University of California at San Francisco (UCSF) cohort, duration of mechanical ventilation (median, 19 hours; IQR, 29 hours), length of intensive care unit stay (median, 5 days; IQR 5 days), duration of any continuous inotropic infusion (median, 66 hours; IQR 72 hours), and preoperative BNP levels (median, $30 \mathrm{pg} / \mathrm{mL}$; IQR, $75 \mathrm{pg} / \mathrm{mL}$ ) were associated with a lower preoperative triceps skin-fold $\mathrm{z}$ score $(P<.05)$. Longer duration of any continuous inotropic infusion and higher preoperative BNP levels were also associated with lower preoperative prealbumin $(12.1 \pm 0.5 \mathrm{mg} / \mathrm{dL})$ and albumin $(3.2 \pm 0.1 ; P<.05)$ levels.
\end{abstract}

Conclusions: Lower total body fat mass and acute and chronic malnourishment are associated with worse clinical outcomes in children undergoing surgery for CHD at UCSF, a resource-abundant institution. There is an inverse correlation between total body fat mass and BNP levels. Duration of inotropic support and BNP increase concomitantly as measures of nutritional status decrease, supporting the hypothesis that malnourishment is associated with decreased myocardial function. (J Thorac Cardiovasc Surg 2014;147:442-50)

\section{Earn CME credits at}

http://cme.ctsnetjournals.org

Critical illness, like many forms of stress, can affect nutritional homeostasis, such as overall energy requirements and caloric intake and losses. ${ }^{1}$ It is well established that

\footnotetext{
$\overline{\text { From Pediatrics, }}{ }^{\mathrm{a}}$ Cardiovascular Research Institute, ${ }^{\mathrm{b}}$ and Surgery, ${ }^{\mathrm{c}}$ University of California, San Francisco, Calif; Unidad de Cirugia Cardiovascular de Guatemala, ${ }^{\mathrm{d}}$ Guatemala City, Guatemala; Surgery ${ }^{\mathrm{e}}$ and Division of Public Health Sciences, ${ }^{\mathrm{f}}$ Washington University, St. Louis, Mo; and Pediatrics, ${ }^{\mathrm{g}}$ Boston Children's Hospital, Boston, Mass.

This research was supported in part by HL61284 (to J.R.F.), HD049303 (to J.R.F.), K23 HL079922 (to R.L.K.), the Pulmonary Hypertension Association (to R.L.K.), the National Institutes of Health (UL RR024131) and the Unidad de Cirugia Cardiovascular de Guatemala.

Disclosures: Authors have nothing to disclose with regard to commercial support.

Received for publication Sept 17, 2012; revisions received Dec 31, 2012; accepted for publication March 15, 2013; available ahead of print April 11, 2013.

Address for reprints: Jeffrey Fineman, MD, University of California at San Francisco, Pediatrics, 505 Parnassus Ave, Box 0106, Room M654, San Francisco, CA 94143 (E-mail: jeff.fineman@ucsf.edu).

$0022-5223 / \$ 36.00$

Copyright (c) 2014 by The American Association for Thoracic Surgery http://dx.doi.org/10.1016/j.jtcvs.2013.03.023
}

malnutrition is associated with poor outcomes in critically ill adults. Contributing factors include malnutritioninduced myocardial dysfunction, vascular endothelial dysfunction, skeletal muscle atrophy, immunosuppression, insulin resistance, and lipolysis. ${ }^{2-5}$ Under the stress of critical illness, malnutrition poses an even greater risk to children because, beyond increased metabolic demand from surgery or disease, there is an increased demand for growth and neurodevelopment. ${ }^{6}$ In fact, malnutrition is one of the most common comorbid conditions in pediatric intensive care units, present in $\sim 20 \%$ of U.S. pediatric intensive care unit admissions. ${ }^{7}$ Several studies demonstrate that the ability of a child to recover after surgery is highly dependent on adequate nutrition. ${ }^{8}$ This is a major factor in children presenting for congenital heart surgery, in which preexisting malnutrition related to cardiac illness, prior hospitalizations, or socioeconomic factors are common. Furthermore, changes in metabolism resulting from surgery are often underestimated. ${ }^{2-5}$ These surgical, stress-induced catabolic responses often result in poor wound healing, myocardial dysfunction, and vascular endothelial dysfunction, and may be exacerbated by poor nutrition. ${ }^{2-5}$ Despite 


\section{Abbreviations and Acronyms \\ BMI = body mass index \\ BNP = B-type natriuretic peptide \\ CHD = congenital heart disease \\ $\mathrm{CI}=$ confidence interval \\ $\mathrm{CPB}=$ cardiopulmonary bypass \\ ICU = intensive care unit \\ LOS = length of stay \\ PCICU = pediatric cardiac intensive care unit \\ UCSF = University of California at San Francisco \\ UNICAR $=$ La Unidad de Cirugia Cardiovascular de Guatemala \\ WHO = World Health Organization \\ TSFZ = triceps skin-fold-for-age $\mathrm{z}$ score}

this, the potential effect of preoperative nutritional status in children with congenital heart disease (CHD) on clinical outcomes remains unclear.

The severity of CHD is linked closely to worse postoperative outcomes. For example, Clancy and colleagues ${ }^{9}$ generated a preoperative risk-of-death prediction model for children undergoing cardiac surgery, demonstrating that cardiac anatomy (single- vs. 2-ventricle repair with or without arch obstruction) was associated highly with postoperative mortality risk. Likewise, the severity of CHD leads to worse nutritional status, particularly when increased pulmonary blood flow, severe cyanosis, and/or pulmonary arterial hypertension are present. ${ }^{10}$ Mechanisms for this association include decreased caloric intake, increased energy expenditure secondary to cardiac failure and/or increased work of breathing, and malabsorption, secondary to poor cardiac output, altered gastrointestinal function, or increased right-sided heart pressure. ${ }^{5}$ However, prospective studies of the relationship between nutritional status and postsurgical outcomes in CHD are lacking.

We sought to assess the impact of preoperative nutritional status on postoperative outcomes in children undergoing surgery for CHD. We hypothesized that poor nutritional status is associated with worse postoperative outcomes in children with CHD. We evaluated the relationship between preoperative anthropometric and laboratory measures of nutritional status and 30-day mortality, intensive care unit (ICU) length of stay (LOS), duration of mechanical ventilation, and duration of continuous inotropic support. To determine the effect of preoperative malnutrition on myocardial performance, we examined the relationship between measures of nutritional status and the duration of inotropic support and preoperative plasma B-type natriuretic peptide (BNP) levels.

\section{METHODS \\ Design and Subjects}

We performed a 2-center, prospective cohort study at the University of California at San Francisco (UCSF), a resource-abundant tertiary pediatric cardiac intensive care unit (PCICU), and La Unidad de Cirugia Cardiovascular de Guatemala (UNICAR), a resource-limited PCICU in Guatemala City. We enrolled 71 children admitted to the PCICU after surgical repair of CHD: 41 at UCSF and 30 at UNICAR. All term children $<5$ years old, male and female, with a Risk Adjustment for Surgery for Congenital Heart Disease Score (RACHS) of $\leq 3$ were eligible for enrollment. We used the RACHS to adjust for baseline risk differences. The RACHS is a well-validated measure of in-hospital mortality risk, with higher scores indicating greater risk (range, 1-6). ${ }^{11}$ We excluded children if they had a RACHS $>3$, a known genetic syndrome, acute and/or chronic extracardiac illnesses, or a hospitalization for $>10$ days prior to surgery exclusively for nutritional support. The a priori restriction of higher RACHS scores was an attempt to achieve comparable surgical severity between study sites.

The study was approved by the institutional review boards at UCSF and UNICAR. Written informed consent was obtained from the guardians of all subjects.

\section{Predictor Variables}

We recorded preoperative anthropometric measures of nutritional status. Specifically, we measured triceps skin fold, a direct measure of peripheral fat mass and an indirect measure of total body fat mass. We used a calibrated Lange skin-fold caliper (Santa Cruz, Calif) by picking up the triceps skin fold between the thumb and forefinger over the posterior surface of the triceps muscle in a vertical line passing upward from the olecranon in the axis of the limb and at the midpoint between the tips of the acromial process and the olecranon. We took measurements while the arm was hanging relaxed at the subject's side. ${ }^{12}$ To minimize intraobserver measurement variation, we applied the jaws 3 times per subject and recorded the mean of the 3 measurements. To minimize interobserver variation, we limited trained observers to 2 at UCSF and 1 at UNICAR. We assigned all subjects a World Health Organization (WHO) triceps skinfold-for-age z score (TSFZ) based on the WHO growth charts. World Health Organization $\mathrm{z}$ scores provide a single international standard that represents the best description of physiologic growth for all children from birth to 5 years of age. ${ }^{13}$

We recorded preoperative laboratory measures of nutritional status, including serum prealbumin, a marker of acute malnourishment, and serum albumin, a marker of chronic malnourishment. Prealbumin, also known as transthyretin, has a half-life of $\sim 2$ days, making it more sensitive than albumin to short-term changes in protein energy status. Its concentration closely reflects recent dietary intake. ${ }^{14}$ Albumin has a serum half-life of approximately 20 days and is a reflection of long-term protein nutritional adequacy. ${ }^{15}$ All preoperative labs were drawn from an indwelling central venous or arterial catheter in the operating room prior to the start of surgery and after $\geq 6$ hours of fasting time. Samples were stored at or below $-30^{\circ} \mathrm{C}$ until the time of processing. Range of freezer time for the samples was 7 to 90 days.

\section{Outcome Variables}

Preoperative outcome. We obtained preoperative plasma BNP levels, a laboratory measure of myocardial stress, with the Triage Meter Plus (Biosite Diagnostic, San Diego, Calif) as described previously. ${ }^{16}$

Postoperative outcomes. We followed all study patients throughout their postoperative course and recorded the following outcomes: 30-day mortality, ICU LOS, duration of mechanical ventilation, and duration of continuous inotropic infusions. There are no strict criteria for the use of mechanical ventilation and inotrope infusions at either institution. However, given the potential deleterious effects of prolonged mechanical ventilation, UCSF institutional practice is to separate patients 
from mechanical ventilation as early in their postoperative course as possible. It must be noted that there are no validated criteria for the discontinuation of mechanical ventilation for patients after surgery for any $\mathrm{CHD}$, and thus the decision to separate a patient from the ventilator was made on an individual basis by the attending physicians, who were blinded to the TSFZ values. Likewise, we chose duration of continuous inotropes as an outcome. At UCSF, patients are generally loaded with milrinone on bypass and, separate from bypass, on a milrinone infusion only, milrinone and dopamine if systemic blood pressure is considered low, and the addition of epinephrine when concerns regarding cardiac output exist. The same general approach is taken at UNICAR with respect to mechanical ventilation. However, inotrope use was significantly different at UNICAR. Usually, patients separated from bypass without inotropes and, when used, dobutamine was often administered in lieu of dopamine, and milrinone was rarely initiated. In addition, inotropes were usually not escalated.

\section{Data Collection and Management}

Study data were collected prospectively and managed using Research Electronic Data Capture electronic data capture tools (Nashville, Tenn) hosted at UCSF. ${ }^{17}$

\section{Statistical Analysis}

We analyzed group differences using the Wilcoxon rank sum test for nonparametric data and the $t$ test for parametric data. We used simple and multivariate logistic regression to study correlations between the continuous predictor variable, TSFZ, and the binary outcome variable, 30-day mortality. Next, we fit simple and multivariate linear regression models to study correlations between the continuous predictor variables (TSFZ, serum prealbumin, and albumin), and continuous clinical outcome variables (ICU LOS, duration of mechanical ventilation, duration of continuous inotropic infusions, and plasma BNP levels).

Multivariate linear regression models were created a priori based on the well-documented risk factors for poor outcomes after pediatric congenital heart surgery (age, gender, cardiopulmonary bypass [CPB] time, and RACHS). ${ }^{11}$ As a result of the relatively limited cohort size, we restricted the number of covariates in these models. ${ }^{18}$ We did not use both predictors of disease severity, CPB and RACHS, in the same model because of colinearity. Cardiopulmonary bypass time was used as a risk predictor for all postoperative outcomes. The RACHS was used as a risk predictor for the outcome, BNP, because this outcome occurred prior to CPB. The addition of age and gender to the majority of the models had little to no material effect on point estimates; however, they were retained for face validity. We stratified all analyses by study site (UCSF and UNICAR), as a result of differences in baseline characteristics. Because all 5 deaths at UNICAR occurred either in the operating room or within a few hours after surgery, they were excluded from the analysis of all postoperative outcomes except 30-day mortality.

\section{Assessment of Regression Model}

We assessed the assumptions inherent in multipredictor linear models. Specifically, we evaluated model linearity, normality, constant variance, and absence of outlying, high-leverage, and influential points. B-type natriuretic peptide was log-transformed, which resulted in an even distribution of the residuals. Statistical analyses were performed using STATA 12 (College Station, Tex), with differences considered significant when 2 -sided $P$ values were $<.05$.

\section{RESULTS}

When comparing patients at UCSF $(n=41)$ and UNICAR $(\mathrm{n}=30)$, there was a trend toward older age at surgery at UNICAR, but this did not reach significance. Patients had significantly lower total body fat mass measured as TSFZ at UNICAR, but the surgical procedures at UCSF were distributed toward higher RACHSs (Table 1). All deaths occurred at UNICAR $(n=5)$. Despite the increased mortality, median ICU LOS and median duration of continuous inotropes were shorter at UNICAR compared with UCSF, suggesting a different postoperative ICU approach with increased disease severity (Table 2). In fact, all deaths occurred either in the operating room or within a few hours of arrival to the ICU, with equivalent ventilator and inotropic support duration.

Although there was no statistically significant association between 30-day mortality and total body fat mass, measured by TSFZ, in the unadjusted and adjusted analysis (unadjusted: odds ratio, 2.7; $P=.28 ; 95 \%$ confidence interval $[\mathrm{CI}], 0.4-16.7$; adjusted for age, gender, and severity of heart disease measured as CPB: odds ratio, $5.2 ; P=.2 ; 95 \% \mathrm{CI}, 0.3-80)$, the positive direction and range of the CI suggests that the existence of a true association between these variables cannot be ruled out. Table 3 shows both the unadjusted and adjusted regression analyses of total body fat mass, measured by TSFZ, and clinical outcomes, stratified by study site. There were no statistically significant relationships at UNICAR $(P<.05)$. However, at UCSF, after adjustment for age, gender, and CPB time, an increase in TSFZ was associated with decreased ICU LOS, decreased duration of mechanical ventilation, decreased duration of dopamine infusion, and decreased duration of milrinone infusion.

As an assessment of the relationship between nutritional status and indicators of myocardial function, we analyzed the relationship between total body fat mass, and serum prealbumin and albumin (indices of acute and chronic malnutrition, respectively) with preoperative plasma BNP and the duration of any inotropic infusion (dopamine, milrinone, epinephrine, norepinephrine, and dobutamine). Before and after adjustment for severity of heart disease (RACHS used for BNP; CPB time used for duration of inotropic infusions), the association between total body fat mass and $\log$ BNP was significant at UCSF, demonstrating a $31 \%$ decrease in BNP level for every 1 unit increase in TSFZ (Table 4, Figure 1). Although the direction of this relationship was the same in the UNICAR group, it did not reach statistical significance. Similarly, after adjustment for age, gender, and CPB time, duration of any continuous inotrope infusion increased with a decrease in TSFZ, paralleling the relationship seen between total body fat mass and BNP (Table 4, Figure 2). Again, results were significant only at UCSF. Before and after adjusting for the RACHS, a higher serum prealbumin level was associated with a decrease in BNP and a decrease in duration of dopamine infusion (Table 4). Last, using the serum albumin level as a marker of chronic malnourishment, we found that, after adjustment for the RACHS, a higher albumin level was associated with a decrease in BNP. There was an overall 
TABLE 1. Baseline characteristics of children

\begin{tabular}{|c|c|c|c|}
\hline Variable & $\mathrm{UCSF}(\mathrm{n}=\mathbf{4 1})$ & $\operatorname{UNICAR}(\mathbf{n}=\mathbf{3 0})$ & $P$ value \\
\hline \multicolumn{4}{|l|}{ Demographic and anthropometric characteristics } \\
\hline Age, months; median (IQR) & $7.3(3.2,47.1)$ & $18(10.2,33)$ & .14 \\
\hline Females, $\mathrm{n}(\%)$ & $24(59)$ & $15(50)$ & .48 \\
\hline TSFZ, median (IQR) & $0.79(-0.1,1.7)$ & $0.21(-0.2,0.7)$ & .03 \\
\hline Prealbumin, $\mathrm{mg} / \mathrm{dL} ;$ mean $\pm \mathrm{SD}$ & $12.1 \pm 3.0$ & $13.9 \pm 5.4$ & .07 \\
\hline Albumin, g/dL; mean $\pm \mathrm{SD}$ & $3.2 \pm 0.4$ & $3.8 \pm 0.4$ & .000 \\
\hline RACHS & & & $.045^{*}$ \\
\hline Score, 1 point, $\mathrm{n}(\%)$ & $4(10)$ & $6(20)$ & \\
\hline $\begin{array}{l}\text { Atrial septal defect surgery (including atrial septal defect secundum, sinus venosus atrial } \\
\text { septal defect, and patent foramen ovale closure) }\end{array}$ & 2 & 5 & \\
\hline Coarctation repair at age $>30 \mathrm{~d}$ & 2 & 1 & \\
\hline Score, 2 points; $\mathrm{n}(\%)$ & $20(49)$ & $18(60)$ & \\
\hline Subaortic stenosis resection & 0 & 2 & \\
\hline Atrial septal defect and ventricular septal defect repair & 3 & 2 & \\
\hline Atrial septal defect primum repair & 1 & 1 & \\
\hline Ventricular septal defect repair & 6 & 9 & \\
\hline Ventricular septal defect closure and pulmonary artery band removal & 1 & 0 & \\
\hline Total repair of tetralogy of Fallot & 3 & 4 & \\
\hline Glenn shunt & 6 & 0 & \\
\hline Coarctation repair at age $\leq 30 \mathrm{~d}$ & 1 & 0 & \\
\hline Score, 3 points; $\mathrm{n}(\%)$ & $17(41)$ & $6(20)$ & \\
\hline Mitral valve replacement & 0 & 1 & \\
\hline Tricuspid valve repositioning for Ebstein anomaly at age $>30 \mathrm{~d}$ & 0 & 1 & \\
\hline Repair of anomalous coronary artery with intrapulmonary tunnel (Takeuchi) & 0 & 2 & \\
\hline Right ventricular-to-pulmonary artery conduit & 3 & 0 & \\
\hline Repair of double-outlet right ventricle with or without repair of right ventricular obstruction & 1 & 0 & \\
\hline Fontan procedure & 7 & 0 & \\
\hline Systemic-to-pulmonary artery shunt & 2 & 0 & \\
\hline Atrial switch operation & 0 & 1 & \\
\hline Arterial switch operation & 3 & 0 & \\
\hline Repair of coarctation and ventricular septal defect closure & 0 & 1 & \\
\hline
\end{tabular}

Statistically significant values are in bold. UCSF, University of California at San Francisco; UNICAR, La Unidad de Cirugia Cardiovascular de Guatemala; IQR, interquartile range; TSFZ, World Health Organization triceps skin-fold-for-age z score; RACHS, Risk Adjustment for Surgery for Congenital Heart Disease Score. *Analysis of variance among groups.

trend in the adjusted analysis toward decreased duration of dopamine requirement with higher serum albumin levels; however, this did not reach statistical significance (Table 4).

\section{DISCUSSION}

In this study, we found that lower total body fat mass as well as acute and chronic indices of malnourishment are associated with worse clinical outcomes in children undergoing surgery for CHD at UCSF. Specifically, the UCSF cohort demonstrated an increase in ICU LOS and duration of mechanical ventilation as anthropometric measures of total body fat mass decreased. Among the UCSF subjects, duration of inotropic support and plasma BNP levels increased concomitantly as measures of nutritional status decreased, supporting the hypothesis that malnourishment is associated with decreased perioperative myocardial function.

Lower weight-for-age and weight-for-height have been reported to be associated with poor outcomes in critical care. Okoromah and colleagues ${ }^{19}$ described a $41 \%$ prevalence of "wasting," described as a WHO weight-for-height $\mathrm{z}$ score of $<-2$, among pediatric cardiac patients in a tertiary teaching hospital in Lagos, Nigeria. Specifically within CHD, malnutrition defined by traditional anthropometry, total body weight and/or plasma protein status, has been linked to increased morbidity and mortality, as indicated by frequent hospitalization, poor surgical outcomes, persistent impairment of somatic growth, and increased death. ${ }^{20}$ Polat and colleagues ${ }^{21}$ found that a lower body mass index (BMI) was associated with impaired postoperative hemodynamic status as well as more long-term failures in gross motor, fine motor, and language skills. Unfortunately, these commonly used anthropometric measures of nutritional status, such as weight-for-length or BMI, are not able to assess the distribution of major components of body weight: fat, lean body mass, and fluid body content. ${ }^{22}$ That is, BMI tends to overestimate body fat in people with increased lean body mass or those who have edema. Conversely, BMI underestimates body fat in people with muscle mass loss, such as children with chronic illness. ${ }^{23}$ 
TABLE 2. Preoperative and postoperative outcomes

\begin{tabular}{lccc}
\hline \multicolumn{1}{c}{ Outcome } & $\begin{array}{c}\text { UCSF } \\
(\mathbf{n = 4 1 )}\end{array}$ & $\begin{array}{c}\text { UNICAR } \\
(\mathbf{n}=\mathbf{3 0})\end{array}$ & $\begin{array}{c}\boldsymbol{P} \\
\text { value }\end{array}$ \\
\hline 30-d mortality, n (\%)* & 0 & $5(17)$ & $\mathbf{. 0 0 7}$ \\
No. of ventilator hours, median (IQR) & $19(11,40)$ & $27(4,96)$ & .5 \\
ICU LOS, days; median (IQR) & $5(3,8)$ & $2(1,10)$ & $\mathbf{. 0 2 4}$ \\
Continuous inotropes & & & \\
Any, hours; median (IQR) & $66(27,99)$ & $0(0,48)$ & $\mathbf{. 0 0 3}$ \\
Dopamine, hours; median (IQR) & $1(0,18)$ & $0(0,27)$ & .7 \\
$\quad$ Milrinone, hours; median (IQR) & $66(25,99)$ & 0 & $<. \mathbf{0 0 1}$ \\
BNP, pg/mL; median (IQR) & $30(14,89)$ & $40(8,290)$ & .8 \\
\hline
\end{tabular}

Statistically significant values are in bold. $U C S F$, University of California at San Francisco; UNICAR, La Unidad de Cirugia Cardiovascular de Guatemala; IQR, interquartile range; ICU LOS, intensive care unit length of stay; $B N P$, B-type natriuretic peptide. *Deaths were excluded from analyses of all clinical outcomes except 30-day mortality.

To our knowledge, no studies have measured the changes in body composition in CHD and their impact on outcomes. In the setting of cardiac disease, it is unreliable to use weight and/or length/height as clinical assessments of nutrition. Total body weight may increase in patients with severe CHD who accumulate large quantities of fluid, thereby masking ongoing cachexia and/or loss of total body fat mass. Previous studies that have relied on BMI as a measure of nutritional status have not addressed the major components of body weight: lean body mass, fat, and fluid, which are particularly pertinent in the pediatric CHD population. Without assessing the distribution of body composition, it becomes difficult to know whether observed clinical outcomes are secondary to edema, cachexia, and/or loss of subcutaneous and visceral fat mass. We believe that our use of TSFZ as a measure of body fat mass is a more valuable measure of true nutritional status because it

TABLE 3. Association between preoperative total body fat mass and postoperative clinical outcomes

\begin{tabular}{|c|c|c|c|c|}
\hline Outcome & $\begin{array}{c}\text { Unadjusted } \\
(95 \% \text { CI })\end{array}$ & $P$ value & $\begin{array}{c}\text { Adjusted } \\
(\mathbf{9 5} \% \mathbf{C I})^{*}\end{array}$ & $P$ value \\
\hline \multicolumn{5}{|c|}{ ICU LOS, days } \\
\hline UCSF & $-3(-6,-1)$ & .04 & $-1.4(-3,-0.1)$ & .04 \\
\hline UNICAR & $-3(-18,13)$ & .8 & $1(-31,32)$ & .9 \\
\hline \multicolumn{5}{|c|}{ Duration of mechanical ventilation, hours } \\
\hline UCSF & $-23(-42,-4)$ & .023 & $-17(-34,-1)$ & .04 \\
\hline UNICAR & $4(-106,115)$ & .9 & $6(-98,110)$ & .9 \\
\hline \multicolumn{5}{|c|}{ Duration of dopamine infusion, hours } \\
\hline UCSF & $-4(-7,-.7)$ & .019 & $-4(-7,-0.8)$ & .015 \\
\hline UNICAR & $-1(-16,14)$ & .9 & $-1(-13,12)$ & .9 \\
\hline \multicolumn{5}{|c|}{ Duration of milrinone infusion, hours } \\
\hline UCSF & $-40(-68,-11)$ & .008 & $-30(-54,-6)$ & .016 \\
\hline UNICAR & $16(-19,52)$ & .3 & $19(-13,51)$ & .2 \\
\hline
\end{tabular}

Statistically significant values are in bold. $C I$, Confidence interval; ICU LOS, intensive care unit length of stay; UCSF, University of California at San Francisco; UNI$C A R$, La Unidad de Cirugia Cardiovascular de Guatemala. *Multiple linear regression for preoperative World Health Organization triceps skin fold-for-age $\mathrm{z}$ score on postoperative clinical outcomes, adjusting for age, gender, and cardiopulmonary bypass time. addresses one major component of total body weight, peripheral fat mass, thereby minimizing the potential for confounding by the accumulation of fluid weight seen commonly in severe heart disease. Although the majority of studies demonstrate that total body fat is associated with skin-fold thickness in obese children, prior studies have presented evidence that this association exists among healthy and underweight children. ${ }^{24,25}$

There have been few studies of the effect of nutritional status on myocardial function. As an indirect determination of myocardial function, we measured preoperative BNP levels and examined the association of these levels with the postoperative inotrope requirement. B-type natriuretic peptide is a 32-amino acid polypeptide hormone produced by the cardiac ventricles, and has become a well-established biomarker in cardiac disease and a routine part of the evaluation of myocardial stress. ${ }^{26}$ Kelleher and colleagues ${ }^{24}$ reported previously that children with hypoplastic left heart syndrome and poorer nutritional status at the time of their stage 1 Norwood procedure demonstrated worse right ventricular function by echocardiogram at the time of their Glenn procedure. Furthermore, perioperative BNP levels have been found to predict outcome-specifically, longer duration of mechanical ventilation and longer ICU stay after surgery for CHD in several disease states. ${ }^{26-31}$

Our study's demonstration of the correlation between poorer nutritional status and increased BNP levels as well as longer duration of inotropic infusions supports prior observations that nutritional status is linked closely to myocardial function. In the UCSF cohort, the children with lower nutritional status and higher BNP levels did not routinely demonstrate clinical evidence of decreased cardiac performance such as low cardiac output syndrome or the need for extracorporeal life support. However, inotropic support was higher in these patients, which could have prevented or masked the clinical manifestations of increased cardiac stress. Although the association between nutrition and BNP levels did not reach statistical significance in the UNICAR cohort $(P>.05)$, the direction of the trend was similar to that seen at UCSF.

In the current study, BNP appears to be a marker of myocardial stress in the setting of childhood malnutrition because the association between preoperative BNP levels and nutritional status mirrors that observed between duration of inotropic infusions and nutritional status. However, multiple recent adult studies have elucidated an inverse relationship between BNP and BMI. This correlation is even stronger when using measures of either fat mass or lean body mass in lieu of BMI. Multiple factors may contribute to this correlation, including increased metabolism of BNP by adipose tissue, inflammatory cytokine levels, endogenous and exogenous alpha-adrenergic agents, and levels of sex hormones. This has been termed 
TABLE 4. Association between preoperative nutritional status and indicators of myocardial performance

\begin{tabular}{|c|c|c|c|c|}
\hline Outcome & Unadjusted $(95 \%$ CI $)$ & $P$ value & Adjusted $(95 \%$ CI) & $P$ value \\
\hline \multicolumn{5}{|c|}{ Association between preoperative total body fat mass and indicators of myocardial function } \\
\hline \multicolumn{5}{|l|}{ BNP, pg/mL* } \\
\hline UCSF & $-47(-77,-17)$ & .004 & $-41(-72,-9)$ & .014 \\
\hline UNICAR & $4(-95,104)$ & .9 & $-5(-68,58)$ & .9 \\
\hline \multicolumn{5}{|c|}{ Any continuous inotropic infusion(s), hours $\dagger, \ddagger$} \\
\hline UCSF & $-40(-68,-11)$ & .008 & $-29(-51,-7)$ & .012 \\
\hline UNICAR & $-16(-79,46)$ & .6 & $-14(-68,40)$ & 6 \\
\hline \multicolumn{5}{|c|}{ Association between preoperative prealbumin levels and indicators of myocardial function } \\
\hline \multicolumn{5}{|c|}{ BNP, pg/mL* } \\
\hline UCSF & $-123(-194,-53)$ & .001 & $-98(-177,-18)$ & .017 \\
\hline UNICAR & $-2(-23,19)$ & .8 & $-3(-17,11)$ & .6 \\
\hline \multicolumn{5}{|c|}{ Duration of dopamine infusion, hours $\dagger$} \\
\hline UCSF & $-3(-5,-1)$ & .002 & $-2(-4,-1)$ & .022 \\
\hline UNICAR & $-1(-3,-1)$ & .4 & $-1(-3,1)$ & .25 \\
\hline \multicolumn{5}{|c|}{ Association between preoperative albumin levels and indicators of myocardial function } \\
\hline \multicolumn{5}{|c|}{ BNP, pg/mL* } \\
\hline UCSF & $-706(-1259,-153)$ & .014 & $-691(-1262,-120)$ & .019 \\
\hline UNICAR & $42(-230,313)$ & .8 & $123(-50,296)$ & .15 \\
\hline \multicolumn{5}{|c|}{ Duration of dopamine infusion, hours $\dagger$} \\
\hline UCSF & $-14(-30,2)$ & .076 & $-11(-25,3)$ & .12 \\
\hline UNICAR & $-26(-54,3)$ & .074 & $-15(-38,9)$ & .2 \\
\hline
\end{tabular}

Statistically significant values are in bold. $C I$, Confidence interval; $B N P$, B-type natriuretic peptide; $U C S F$, University of California at San Francisco; UNICAR, La Unidad de Cirugia Cardiovascular de Guatemala. *Multiple linear regression for preoperative measures of nutritional status (World Health Organization triceps skin fold-for-age $\mathrm{z}$ score, prealbumin level, and albumin level) on preoperative plasma BNP level, adjusting for age, gender, and Risk Adjustment for Surgery for Congenital Heart Disease Score. $\dagger$ Multiple linear regression for preoperative measures of nutritional status (World Health Organization triceps skin fold-for-age z score, prealbumin level, albumin level) on duration of inotropic infusion(s), adjusting for age, gender, and cardiopulmonary bypass time. †๋ Includes dopamine, milrinone, epinephrine, norepinephrine, and dobutamine.

the natriuretic handicap, and suggests that BNP levels may not be a reliable biomarker of myocardial performance in the setting of severe nutritional abnormalities. ${ }^{32}$ However, the focus has been uniformly on adult obesity, with no investigation of this correlation among children with
CHD. Because the response of the cardiac endocrine system is the product of several, often opposing, physiologic interactions, this potential relationship becomes an important consideration when faced with the task of interpreting BNP levels in the clinical setting of moderate to severe

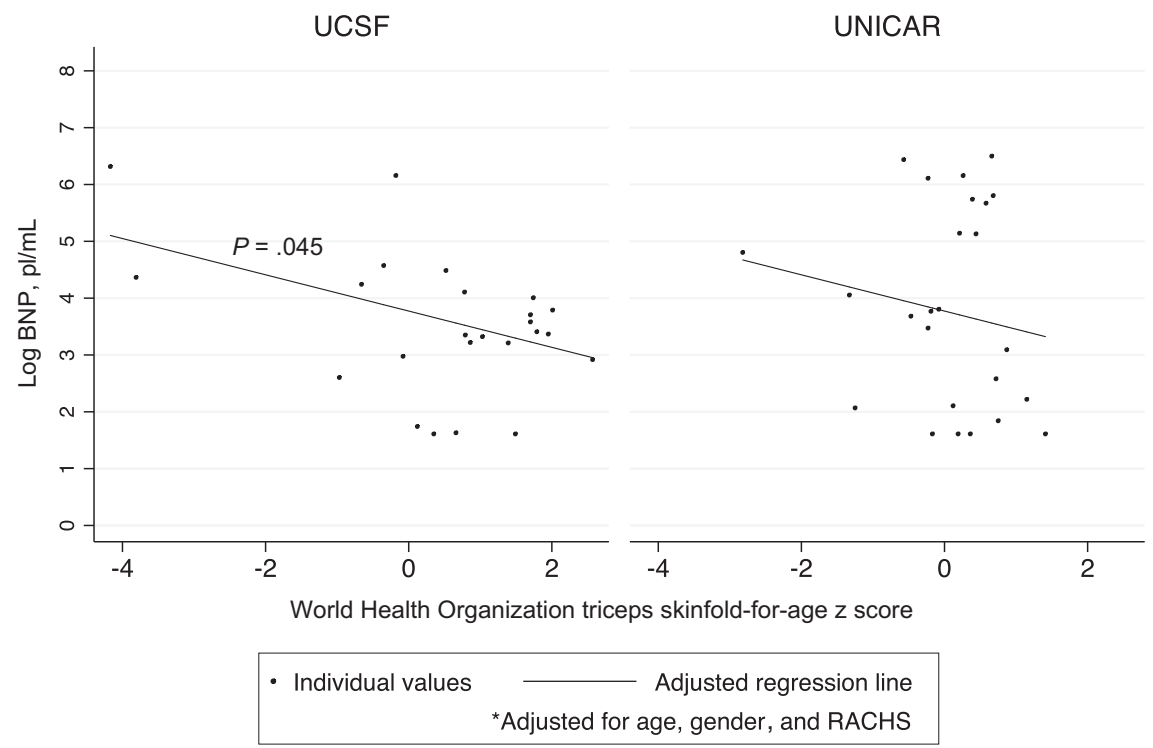

FIGURE 1. Association between total body fat mass (World Health Organization triceps skin-fold-for-age z score) and log-transformed preoperative plasma B-type natriuretic peptide $(B N P)$ levels at the University of California at San Francisco $(U C S F ; \mathrm{n}=41)$ and La Unidad de Cirugia Cardiovascular de Guatemala (UNICAR; $\mathrm{n}=30$ ) after adjustment for age, gender, and Risk Adjustment for Surgery for Congenital Heart Disease Score $(R A C H S)$. $P=.045$. 


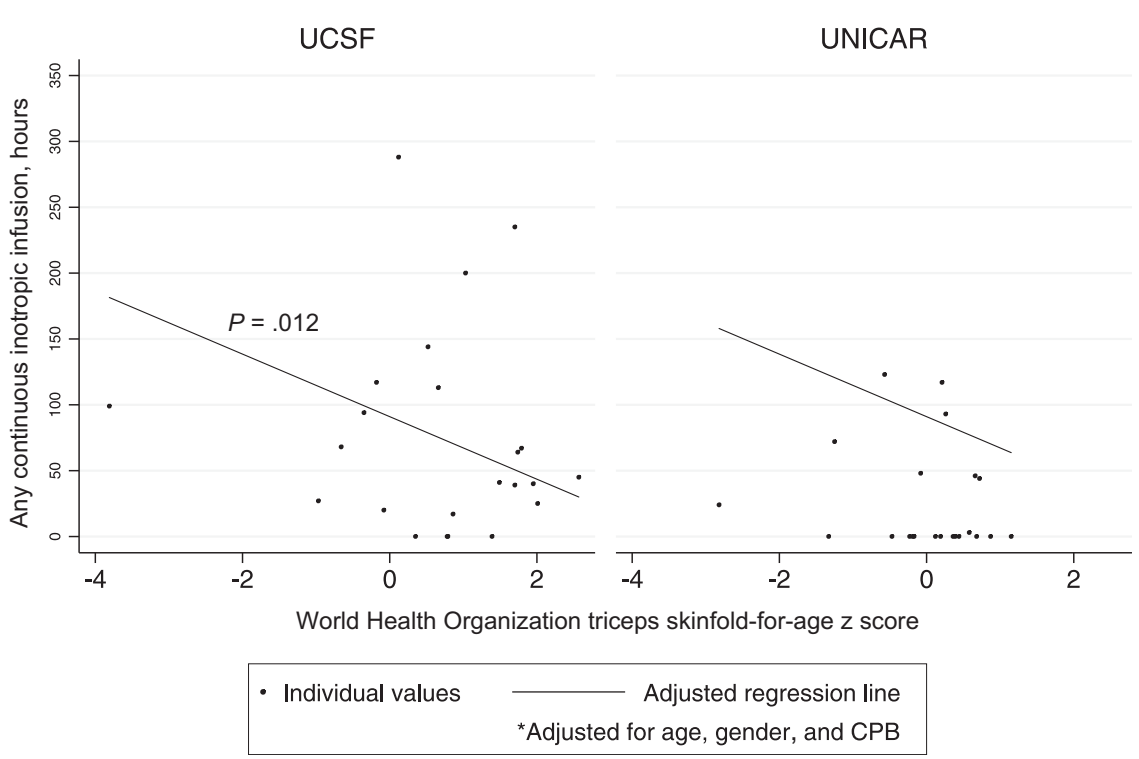

FIGURE 2. Association between total body fat mass (World Health Organization triceps skin-fold-for-age $\mathrm{z}$ score) and postoperative duration of any continuous inotropic infusions at the University of California at San Francisco $(U C S F ; \mathrm{n}=41)$ and La Unidad de Cirugia Cardiovascular de Guatemala (UNI$C A R ; \mathrm{n}=30)$ after adjustment for age, gender, and cardiopulmonary bypass $(C P B)$ time. $P=.012$.

malnutrition or obesity. Therefore, the relationship between anthropometric measures and BNP warrant further study.

There were several important differences noted between the UCSF and UNICAR study populations. For example, the associations between outcomes and nutritional status (lower TSFZ, acute and chronic malnourishment) were strikingly stronger at UCSF compared with UNICAR. We believe that, by fitting our multivariate models with either the RACHS or CPB time as predictors, we minimized adequately the potential for an effect-cause phenomenon (ie, the outcome causes the predictor). That is, the model was constructed to isolate the impact of nutrition on postoperative outcomes, independent of the severity of heart disease. Weaker associations between nutritional status and outcomes in the UNICAR cohort may be related, in large part, to the differential approach to inotrope use and the lack of escalation of care as patients' clinical status worsened. Despite increased 30-day mortality and documented evidence of low cardiac output syndrome and capillary leak syndrome in multiple patients, the treatment of these patients was not intensified with new and/or additional continuous inotropes, delay of extubation, reintubation, and/or prolonged ICU LOS. ${ }^{33}$ This decreased variability in clinical outcomes in the UNICAR cohort may have influenced the findings. As a result of the decreased accessibility of care, it is possible that severely malnourished patients with CHD die prior to presenting to the hospital. Similarly, because ICU LOS and interventions were not altered by severity of clinical status, it is possible that many patients die earlier after hospital discharge. We speculate that more long-term outcomes are superior measures of the effects of nutritional status after surgery for CHD in resource-limited populations, but this warrants further study.

The inclusion of 2 very different sites is crucial because it demonstrates how different populations may have different responses to malnourishment. The reasons for these different responses could be embedded in differences in several factors, including genetics, diet, and micronutrient status. For example, it is possible that other unmeasured factors, such as micronutrient status, play a crucial role in the endocrine response to myocardial dysfunction in the setting of malnutrition. Last, center-specific practice styles and/or surgical styles may play a role in the difference in results by study center. Regardless of the underlying causes of the differing associations between cohorts, our data support the idea that a successful nutrition-based intervention in the CHD population at one site may not be successful at another site.

There are limitations to our study that merit discussion. Although this is a pilot study, the small sample size was a limiting factor in interpretation of associations between nutritional status and outcomes, particularly because of the loss of power incurred by our stratification by study site and fewer outcomes at UNICAR. Specifically, although the statistical analysis of the association between 30-day mortality and total body fat mass at UNICAR yielded a statistically nonsignificant $P$ value of .2 , the effect size of 5.2 combined with the markedly positive $95 \% \mathrm{CI}$, raises the question of whether a true association between these factors exists that our study was not powered adequately to detect. In addition, some of the significant associations had relatively weak correlations, making it reasonable to interpret the results cautiously. Furthermore, the lack of 
a large number of severely malnourished subjects in either cohort further reduced the statistical power of the study.

The cardiac population is the most homogeneous group of patients in a pediatric ICU setting. However, there was a wide range of congenital heart lesions included in the study. We attempted to adjust for severity of heart disease with the RACHSs and CPB time. However, there are inherent physiologic differences between right-to-left shunting lesions and left-to-right shunting lesions. For example, we know that the amount of pulmonary blood flow is associated with postsurgical outcomes. ${ }^{34}$ If, in fact, the amount of pulmonary blood flow has a causal relationship to nutritional status, these differences could constitute an unmeasured confounder in this study. Unfortunately, given the small sample size, adjustments in baseline physiologic parameters could not be attempted.

Last, although TSFZ is superior to BMI, it has limitations. Specifically, this method only measures one type of fat: subcutaneous adipose tissue (fat under the skin). Two individuals might have nearly identical measurements at all the skin-fold sites, yet differ greatly in their body fat levels as a result of differences in other body fat deposits such as visceral adipose tissue (fat in the abdominal cavity). There is a need for studies using 4-compartment techniques that allow for the measurement of fat, fat-free mass, bone mineral content, total body water, extracellular water, total adipose tissue and its deposits (visceral, subcutaneous, and intermuscular), skeletal muscle, select organs, and ectopic fat deposits. Clinicians can quantify a number of body components and, with longitudinal assessment, can track changes in health and disease with implications for understanding efficacy of nutritional and clinical interventions, diagnosis, prevention, and treatment in clinical settings. ${ }^{23,32}$

In summary, we describe the first prospective study that investigated concomitantly the effect of nutritional status on clinical outcomes in children undergoing surgery for CHD in 2 differing subpopulations: (1) UCSF, a resourceabundant cohort, and (2) UNICAR, a resource-limited cohort. In this design, we demonstrate robustly the subpopulation-based variability that exists in the associations between preoperative nutritional status and perioperative outcomes. In addition to resource availability, we speculate that this expected variability was a result, in part, of differences in clinical practice norms. We observed that lower total body fat mass as well as acute and chronic malnourishment were associated with worse clinical outcomes and indices of myocardial performance in children undergoing surgery for $\mathrm{CHD}$. These results suggest that clinicians and surgeons should consider carefully a child's nutritional status when planning nonemergency surgery for CHD and when counseling parents about potential risks of surgery. Specifically, the observed increase in postoperative complications is worrisome because of its potential detrimental effects on long-term growth and neurodevelopment. The potential increased costs of this subpopulation of children with CHD is concerning as well. These findings, in conjunction with previous observations of longer hospital stays and higher rates of readmissions among malnourished children undergoing surgery for CHD, support the notion that efforts to facilitate effective preoperative interventions to improve nutrition and patient outcomes are warranted..$^{35}$ The implications for patient outcomes and safety related to nutrition merit continued evaluation.

The authors thank the pediatric critical care fellows, the Pediatric Clinical Research Center, the cardiac intensive care nurses, Ximena Garcia Cruz, Cesar Najera, Christine Sun, Wenhui Gong, MD, PhD, Stephen Black, PhD, and Martina Steurer-Muller, MD, for their invaluable assistance with the study.

\section{References}

1. Singh N, Gupta D, Aggarwal AN, Agarwal R, Jindal SK. An assessment of nutritional support to critically ill patients and its correlation with outcomes in a respiratory intensive care unit. Respir Care. 2009;54:1688-96.

2. Barton JS, Hindmarsh PC, Scrimgeour CM, Rennie MJ, Preece MA. Energy expenditure in congenital heart disease. Arch Dis Childhood. 1994;70:5-9.

3. Cameron JW, Rosenthal A, Olson AD. Malnutrition in hospitalized children with congenital heart disease. Arch Pediatr Adolesc Med. 1995;149:1098-102.

4. Jeejeebhoy KN. Nutritional assessment. Nutrition. 2000;16:585-90.

5. Mehta NM, Duggan CP. Nutritional deficiencies during critical illness. Pediatr Clin North Am. 2009;56:1143-60.

6. Udani S. Feeding in the PICU. Indian J Pediatr. 2001;68:333-7.

7. Hulst J, Joosten K, Zimmermann L, Hop W, van Buuren S, Büller H, et al. Malnutrition in critically ill children: from admission to 6 months after discharge. Clin Nutr. 2004;23:223-32.

8. Pollack MM, Ruttimann UE, Wiley JS. Nutritional depletions in critically ill children: associations with physiologic instability and increased quantity of care. J Parenter Enter Nutr. 1985;9:309-13.

9. Clancy RR, McGaurn SA, Wernovsky G, Spray TL, Norwood WI, Jacobs ML, et al. Preoperative risk-of-death prediction model in heart surgery with deep hypothermic circulatory arrest in the neonate. J Thorac Cardiovasc Surg. 2000; 119:347-57

10. Varan B, Tokel K, Yilmaz G. Malnutrition and growth failure in cyanotic and acyanotic congenital heart disease with and without pulmonary hypertension. Arch Dis Childhood. 1999;81:49-52.

11. Jenkins KJ, Gauvreau K, Newburger JW, Spray TL, Moller JH, Iezzoni LI. Consensus-based method for risk adjustment for surgery for congenital heart disease. J Thorac Cardiovasc Surg. 2002;123:110-8.

12. Tanner JM, Whitehouse RH. Revised standards for triceps and subscapular skinfolds in British children. Arch Dis Childhood. 1975;50:142-5.

13. WHO Multicentre Growth Reference Study Group. Assessment of differences in linear growth among populations in the WHO Multicentre Growth Reference Study. Acta Paediatr Suppl. 2006;450:56-65.

14. Mears E. Outcomes of continuous process improvement of a nutritional care program incorporating serum prealbumin measurements. Nutrition. 1996;12: 479-84.

15. Shenkin A, Cederblad G, Elia M. Laboratory assessment of protein-energy status. Clin Chim Acta. 1996;253:S5-59.

16. Hsu J-H, Oishi PE, Keller RL, Chikovani O, Karl TR, Azakie A, et al Perioperative B-type natriuretic peptide levels predict outcome after bidirectional cavopulmonary anastomosis and total cavopulmonary connection. J Thorac Cardiovasc Surg. 2008;135:746-53.

17. Harris PA, Taylor R, Thielke R, Payne J, Gonzalez N, Conde JG. Research electronic data capture (REDCap): a metadata-driven methodology and workflow process for providing translational research informatics support J Biomed Informatics. 2009;42:377-81. 
18. Vittinghoff E, Glidden DV, Shiboski SC, McCulloch CE. Regression methods in biostatistics: linear, logistic, survival, and repeated measures models. New York, NY: Springer; 2011.

19. Okoromah CAN, Ekure EN, Lesi FEA, Okunowo WO, Tijani BO, Okeiyi JC. Prevalence, profile and predictors of malnutrition in children with congenital heart defects: a case-control observational study. Arch Dis Childhood. 2011;96:354-60.

20. Freeman LM, Roubenoff R. The nutrition implications of cardiac cachexia. Nutr. Rev. 1994;52:340-7.

21. Polat S, Okuyaz C, Hallioğlu O, Mert E, Makharoblidze K. Evaluation of growth and neurodevelopment in children with congenital heart disease. Pediatr Int. 2011;53:345-9.

22. Lee SY, Gallagher D. Assessment methods in human body composition. Curr Opin Clin Nutr Metab Care. 2008;11:566-72.

23. Shirley S, Davis LL, Carlson BW. The relationship between body mass index/body composition and survival in patients with heart failure. J Am Acad Nurse Pract. 2008;20:326-32.

24. Kelleher DK, Laussen P, Teixeira-Pinto A, Duggan C. Growth and correlates of nutritional status among infants with hypoplastic left heart syndrome (HLHS) after stage 1 Norwood procedure. Nutrition. 2006;22:237-44.

25. Hsu J-H, Keller RL, Chikovani O, Cheng H, Hollander SA, Karl TR, et al. B-type natriuretic peptide levels predict outcome after neonatal cardiac surgery. J Thorac Cardiovasc Surg. 2007;134:939-45.

26. Doust JA. A systematic review of the diagnostic accuracy of natriuretic peptides for heart failure. Arch Intern Med. 2004;164:1978-84.

27. Hsu C-P, Lin S-J, Chung M-Y, Lu T-M. Asymmetric dimethylarginine predicts clinical outcomes in ischemic chronic heart failure. Atherosclerosis. 2012;225:504-10.
28. Cannesson M, Bionda C, Gostoli B, Raisky O, di Filippo S, Bompard D, et al. Time course and prognostic value of plasma B-type natriuretic peptide concentration in neonates undergoing the arterial switch operation. Anesth Analg. 2007;104:1059-65.

29. Shih C-Y, Sapru A, Oishi P, Azakie A, Karl TR, Harmon C, et al. Alterations in plasma B-type natriuretic peptide levels after repair of congenital heart defects: a potential perioperative marker. J Thorac Cardiovasc Surg. 2006;131: 632-8.

30. Chikovani O, Hsu J-H, Keller R, Karl TR, Azakie A, Adatia I, et al. B-type natriuretic peptide levels predict outcomes for children on extracorporeal life support after cardiac surgery. J Thorac Cardiovasc Surg. 2007;134: 1179-87.

31. Amirnovin R, Keller RL, Herrera C, Hsu J-H, Datar S, Karl TR, et al. B-type natriuretic peptide levels predict outcomes in infants undergoing cardiac surgery in a lesion-dependent fashion. J Thorac Cardiovasc Surg. 2013;145: 1279-87.

32. Clerico A, Giannoni A, Vittorini S, Emdin M. The paradox of low BNP levels in obesity. Heart Fail Rev. 2012;17:81-96.

33. Hoffman TM. Efficacy and safety of milrinone in preventing low cardiac output syndrome in infants and children after corrective surgery for congenital heart disease. Circulation. 2003;107:996-1002.

34. Rodefeld MD, Hanley FL. Neonatal truncus arteriosus repair: surgical techniques and clinical management. Semin Thorac Cardiovasc Surg Pediatr Card Surg Аппи. 2002;5:212-7.

35. Forchielli ML, McColl R, Walker WA, Lo C. Children with congenital heart disease: a nutrition challenge. Nutr Rev. 1994;52:348-53. 